\title{
Aqueous misdirection syndrome: an interesting case presentation
}

This article was published in the following Dove Press journal:

Clinical Ophthalmology

29 January 2015

Number of times this article has been viewed

\section{Prima Moinul' \\ Cindy ML Hutnik ${ }^{2}$}

'Faculty of Medicine, University of Calgary, Calgary, AB, Canada; 'Ivey Eye Institute, St Joseph's Health Care, Department of Ophthalmology, University of Western Ontario, London, ON, Canada
Correspondence: Cindy ML Hutnik Ivey Eye Institute, St Joseph's Health Care, Department of Ophthalmology, University of Western Ontario,

268 Grosvenor Street, London,

Ontario N6A 4V2, Canada

$\mathrm{Tel}+\mathrm{I} 5196466272$

Fax +I 5196466410

Email cindy.hutnik@sjhc.london.on.ca
Objective: To report a case of an aqueous misdirection-like presentation in a pseudophakic patient.

Design: Retrospective case review.

Participant: An 84-year-old pseudophakic gentleman presented with bilateral blurred vision 8 years after cataract surgery. A refractive shift with shallow anterior chambers and elevated intraocular pressures were noted. No corneal edema was noted. Although aqueous suppression and topical atropine would relieve the signs and symptoms, the effect was temporary with fluctuating and variable changes in refraction, anterior chamber depth, and intraocular pressure. The presence of patent iridotomies had no effect on the fluctuations. A pars plana vitrectomy and surgical iridectomy were successful in preventing further fluctuations.

Conclusion: Aqueous misdirection is a form of secondary angle closure glaucoma marked by elevated intraocular pressures, myopic shift in refraction, and central shallowing of the anterior chamber. Here, a case of a pseudophakic patient experiencing bilateral and fluctuating signs and symptoms resembling aqueous misdirection is presented. Surgical intervention with a pars plana vitrectomy and iridectomy prevented further fluctuations.

Keywords: aqueous misdirection, glaucoma, pars plana vitrectomy, secondary angle closure

\section{Introduction}

Aqueous misdirection, also known as malignant glaucoma, is a term coined by von Graefe in 1869 to describe an uncommon, but serious condition that presents typically following incisional ocular surgeries, such as iridectomy or filtering surgery. ${ }^{1}$ It may also present following phacoemulsification, cyclophotocoagulation, neodymium:yttriumaluminum-garnet, laser capsulotomy, and in some cases, even outside procedural settings in phakic and pseudophakic eyes. ${ }^{2}$ Aqueous misdirection is characterized by a shallow central and peripheral anterior chamber (AC), forward displacement of the lens-iris diaphragm, and normal or elevated intraocular pressure (IOP). ${ }^{3}$ This anterior displacement of the lens induces a myopic shift in refraction, resulting in blurry vision in previously emmetropic patients. Although aqueous misdirection is prevalent in $0.4 \%-6 \%$ of postsurgical cases, patients with a history of hyperopia, previous angle closure, micro- or nanophthalmos, or partially closed angles during surgery can be predisposed to a greater risk. ${ }^{4}$

The exact mechanism by which aqueous misdirection occurs is still poorly understood. However, the historical belief is that an abnormal anatomic relationship exists between the ciliary bodies, lens, and anterior hyaloid causing diversion of fluid into the posterior chamber. ${ }^{5}$ Aqueous fluid builds within the vitreous body and raises IOP, and thereby, exerts a force on the anterior hyaloid that causes a forward displacement of the lens-iris diaphragm. ${ }^{6}$ Ultrasound biomicroscopy studies also provide evidence 
by illustrating that these eyes display supraciliary fluid accumulation that pushes anteriorly rotated ciliary processes against the lens equator. ${ }^{7}$ Whether this anatomical configuration of ciliary processes lead to diversion of fluid or is itself an outcome of volume expansion is unclear.

In normal eyes, aqueous humor flows freely from the posterior to the $\mathrm{AC}$, equalizing the pressure across the lens-iris diaphragm. However, in aqueous misdirection, there is considerable resistance to aqueous flow at the anterior hyaloid that aggravates the condition by fueling a vicious cycle of continued aqueous production, entrapment of fluid, and pressure elevation. ${ }^{8}$ The gradual pressure differential leads to narrowing of the AC, which may progress to complete angle closure if left untreated. These changes may develop immediately following surgery, after discontinuation of cycloplegics or initiation of miotics, or many years after the insult. ${ }^{9}$ Interestingly, the incidence of aqueous misdirection in one eye increases the risk of occurrence in the fellow eye, regardless of any history of glaucoma. ${ }^{9}$ As a result, prophylactic treatment including cycloplegics, peripheral iridotomy, IOP control, and continued monitoring is necessary to avoid excessive shallowing of angles in both eyes.

More recently, Dr Harry Quigley suggested an alternate explanation for the clinical findings. ${ }^{10}$ He postulated that expansion of the choroid raises IOP and creates a pressure differential between the posterior and anterior chamber that leads to forward displacement of the lens-iris diaphragm and narrowing of the angle. ${ }^{10,11}$ Other theories, by Shaffer and Hoskins, suggest that pooling of aqueous humor posterior to the vitreous causes anterior displacement of lens, ciliary, and iris. ${ }^{12}$ Because the mechanism is not fully elucidated, there is no single, optimal treatment.

Consequently, management of aqueous misdirection can be challenging. Pharmacotherapy is typically first-line treatment involving administration of cycloplegics to tighten the lens zonules and pull the anteriorly displaced lens back. ${ }^{13}$ Aqueous suppressants (specifically, beta-blockers, alpha adrenergic agonists, or carbonic anhydrase inhibitors) and hyperosmotics are administered to facilitate management and lower IOP. ${ }^{14}$ However, medical management can be curative in only half of the patients..$^{15}$ Patients refractory to medical therapy may require laser treatment with neodymium:yttrium-aluminum-garnet, laser capsulotomy, laser hyaloidotomy, or cyclodiode laser photocoagulation. ${ }^{16-18}$ It is believed that large optic lens or synechiae formed between the intraocular lens (IOL) and capsule may also act as a barrier to anterior flow of aqueous. In these cases, laser capsulotomy creates a patent passageway for fluid flow from posterior to the AC. ${ }^{18}$ If patients continue to be irresponsive, invasive surgical procedures can augment treatment by disrupting the anterior hyaloid face to create a permanent passageway for aqueous flow. ${ }^{4}$ These procedures may include vitrectomy with iridozonulectomy, hyalozonulectomy, or a complete vitrectomy-iridectomy-zonulectomy. ${ }^{15}$ Many studies have reported that successful management is ultimately achieved with a combination of numerous techniques; but there is still a lack of consensus about the most optimal therapeutic strategy.

In conclusion, the management of aqueous misdirection continues to be a therapeutic challenge attributed mainly to our limited understanding of its pathophysiology. Recognizing that the condition may have a recurrent and fluctuating presentation is important for long-term monitoring and management.

\section{Case study}

An 84-year-old Caucasian pseudophakic male with a previously stable refraction presented with sudden onset of bilateral blurry vision and best-corrected visual acuities no better than 20/30 in either eye. The ACs were noted to be shallower compared to previous assessments with appositional closure. The IOP was $13 \mathrm{mmHg}$ bilaterally. Eight years previously he had undergone an uneventful phacoemulsification cataract extraction and placement of single-piece acrylic IOL implants. Medical and family history were otherwise unremarkable.

Anterior segment examination and ultrasound biomicroscopy demonstrated an anterior displacement of the IOL implants with contact between the IOL implants and iris. A myopic shift was also noted. Central corneal thicknesses were 490 microns in both the eyes with no evidence of corneal pathology. Laser peripheral iridotomies resulted in deepening of the chamber and angles and a refractive shift back to emmetropia.

Two years later, the patient returned with symptoms of bilateral blurred vision. The IOP in the left eye was measured to be $30 \mathrm{mmHg}$ with more than 240 degrees of angle not visible on gonioscopy. IOP in the right eye was in the mid-teens. The bilateral shallowing of the AC was noted with myopic shifts and best-corrected visual acuities of 20/70 and 20/50 in the right and left eyes, respectively. Peripheral iridotomies remained patent. The left eye was treated with atropine $1 \%$ and three classes of aqueous suppressants, as well as latanoprost. Atropine 1\% was also used in the right eye. Within 2 weeks, IOP normalized to $9 \mathrm{mmHg}$ and $11 \mathrm{mmHg}$ in the right and left eyes, respectively, the chambers/angles 
deepened and the refraction returned to emmetropia. At this time, the patient was kept on one IOP-lowering agent and atropine to mitigate against potential future IOP spikes. Despite this, a recurrence of the signs and symptoms occurred 1 month later with the best-corrected visual acuity of 20/40 in the right eye (OD) (IOP $14 \mathrm{mmHg}$ ) and 20/100 in the left eye (OS) (IOP $29 \mathrm{mmHg}$ ). Corneal edema was never noted at any time. Repeat ultrasound biomicroscopy revealed very narrow angle configurations with no evidence of choroidal effusions. Gonioscopy demonstrated appositional closure of the angles with patent iridotomies.

Due to the fluctuating and recurrent nature of the findings despite patent iridotomies and medical therapy, a pars plana vitrectomy (PPV) combined with a surgical iridectomy was carried out in the left eye. Anterior hyaloidectomy was also performed during this time. At the 3-month postoperative follow-up, the patient's best-corrected visual acuity improved slightly (20/40-1 OD and 20/40 OS), and IOPs again stabilized to normal limits (14 $\mathrm{mmHg}$ OD and $16 \mathrm{mmHg}$ OS) on a single aqueous suppressant. Once the left eye was stabilized, the right eye also underwent a PPV with surgical iridectomy. Although some fluctuation was noted postvitrectomy at 6 months following surgery, no further episodes were noted up to 4 years later. The patient was continued on a single aqueous suppressant with a stable refraction and IOPs in the teens. Visual fields and optic disks were stable throughout his entire clinical course. He continues to be followed up every 6 months and has been advised to call immediately should any change in symptoms occur.

\section{Discussion}

Aqueous misdirection syndrome continues to be a therapeutic challenge. To our knowledge, there are limited reported cases about treatment of recurrent symptoms. Here, we highlighted a case of recurrent aqueous misdirection-like presentation that was treated successfully by PPV, augmented with laser and pharmacotherapy. Although the patient initially presented with normal IOPs (13 mmHg bilaterally) and clear corneas, the presence of anterior displacement of the lens-iris diaphragm and a myopic shift in refraction without suprachoroidal hemorrhage or effusion were suggestive of aqueous misdirection syndrome. Subsequent temporary return to emmetropia and deepening of the AC, mainly after laser iridotomy and topical atropine treatment, followed by another symptomatic attack in the presence of patent iridotomies also support the diagnosis. Literature indicates that successful management of recalcitrant cases is achieved mainly after disruption of the anterior hyaloid. ${ }^{19}$ PPV was performed in this case to debulk the vitreous and hyaloidectomy reset the lens-iris diaphragm, which resulted in an increase in the AC depth and thereby, reduced the risk of corneal endothelial injury. ${ }^{20}$ As anticipated in an aqueous misdirection case, the fluctuant pattern of recurrent symptoms ceased only after hyaloidectomy. ${ }^{21,22}$ As a result, we were able to taper the patient's drops to a single drop after surgery.

The occurrence of aqueous misdirection in one eye significantly increases the risk to the fellow eye. ${ }^{23}$ However, the rare incidences of simultaneous bilateral presentations have only been documented in few reports. ${ }^{24}$ In our case, it was interesting to note that despite bilateral shallowing and changes in refraction, elevated IOP was documented in only one eye during relapse. We suspect the left eye presented first, due to changes observed in gonioscopy, and increased imminent risk to the contralateral eye. The most responsible pathophysiology can be attributed to the establishment of a pressure gradient between the posterior vitreous cavity and cerebral spinal fluid at the retrobulbar space. The pressure gradient with histologic changes in the lamina cribrosa causes movement of fluid in the subarachnoid space of the optic nerve, contributing to elevated cerebral spinal fluid pressures. ${ }^{25}$ Bidirectional flow of cerebral spinal fluid facilitates further fluid migration to the subarachnoid space of the contralateral eye, causing displacement of the vitreous body anteriorly. ${ }^{24,26}$ This force on the lens-iris diaphragm displaces the IOL and creates a myopic shift in vision. Holistically in this study, the visual and structural changes refractory to medical and laser treatments were more suggestive of an aqueous misdirection-like appearance than changes in IOP. This is also supported by case reports documenting aqueous misdirection development in eyes with normal IOPs days to years following procedures. ${ }^{18,23}$ Although it is difficult to postulate the etiology of spontaneous aqueous misdirection-like presentation 8 years postoperatively, it is suspected that, previous surgeries, anatomic predisposition, increased resistance at the trabecular meshwork, aging, and smaller eyes can be contributory. ${ }^{22,27}$

In conclusion, the goal of our treatment is to establish a permanent passage between the posterior and ACs for fluid drainage in eyes refractory to laser and pharmacotherapy. Although making the eye unicameral lowers IOP and helps to deepen the AC, maintenance treatment with pharmacotherapy may be necessary for some patients for different lengths of time afterwards. It is also important to note that the risk to the fellow eye is significantly elevated in aqueous misdirection; and thus, prophylactic treatment with aqueous suppressants and peripheral iridotomy must be considered. Careful follow-up and continued monitoring of patients with aqueous misdirection 
ensures optimal care and better prognosis. In the future, comparative therapeutic studies in multicenter, long-term trials are warranted to better understand surgical techniques and confirm findings.

\section{Disclosure}

Descriptive, printed copies of the surgical techniques have not been published previously. The authors have full access to the data within this manuscript and take full responsibility for the integrity of the data/data analysis. The authors have no financial and/or proprietary interests to declare.

\section{References}

1. Brubaker L. Conflict of interest: what is the role of our professional societies? Neurourol Urodyn. 2012;31(8):1217-1218.

2. Saunders PP, Douglas GR, Feldman F, Stein RM. Bilateral malignant glaucoma. Can J Ophthalmol. 1992;27(1):19-21.

3. Greenfield DS, Tello C, Budenz DL, Liebmann JM, Ritch R. Aqueous misdirection after glaucoma drainage device implantation. Ophthalmology. 1999;106(5):1035-1040.

4. Shahid H, Salmon JF. Malignant glaucoma: a review of the modern literature. J Ophthalmol. 2012;2012:852659.

5. Byrnes GA, Leen MM, Wong TP, Benson WE. Vitrectomy for ciliary block (malignant) glaucoma. Ophthalmology. 1995;102(9):1308-1311.

6. Epstein DL. Pseudophakic malignant glaucoma - is it really pseudomalignant? Am J Ophthalmol. 1987;103(2):231-233.

7. Tello C, Chi T, Shepps G, Liebmann J, Ritch R. Ultrasound biomicroscopy in pseudophakic malignant glaucoma. Ophthalmology. 1993; 100(9):1330-1334.

8. Fatt I. Hydraulic flow conductivity of the vitreous gel. Invest Ophthalmol Vis Sci. 1977;16(6):565-568.

9. Ruben S, Tsai J, Hitchings RA. Malignant glaucoma and its management. Br J Ophthalmol. 1997;81(2):163-167.

10. Quigley HA. Angle-closure glaucoma-simpler answers to complex mechanisms: LXVI Edward Jackson memorial lecture. Am J Ophthalmol. 2009;148(5):657-669. e1.

11. Quigley HA, Friedman DS, Congdon NG. Possible mechanisms of primary angle-closure and malignant glaucoma. J Glaucoma. 2003;12(2): $167-180$.

12. Shaffer RN, Hoskins HD Jr. Ciliary block (malignant) glaucoma. Ophthalmology. 1978;85(3):215-221.
13. Chandler PA, Grant WM. Mydriatic-cycloplegic treatment in malignant glaucoma. Arch Ophthalmol. 1962;68:353-359.

14. Lockie P. Ciliary-block glaucoma treated by posterior capsulotomy. Aust N Z J Ophthalmol. 1987;15(3):207-209.

15. Debrouwere V, Stalmans P, Van Calster J, Spileers W, Zeyen T, Stalmans I. Outcomes of different management options for malignant glaucoma: a retrospective study. Graefes Arch Clin Exp Ophthalmol. 2012;250(1):131-141.

16. Epstein DL, Steinert RF, Puliafito CA. Neodymium-YAG laser therapy to the anterior hyaloid in aphakic malignant (ciliovitreal block) glaucoma. Am J Ophthalmol. 1984;98(2):137-143.

17. Stumpf TH, Austin M, Bloom PA, McNaught A, Morgan JE. Transscleral cyclodiode laser photocoagulation in the treatment of aqueous misdirection syndrome. Ophthalmology. 2008;115(11):2058-2061.

18. Muqit MM, Menage MJ. Malignant glaucoma after phacoemulsification: treatment with diode laser cyclophotocoagulation. $J$ Cataract Refract Surg. 2007;33(1):130-132.

19. Bitrian E, Caprioli J. Pars plana anterior vitrectomy, hyaloidozonulectomy, and iridectomy for aqueous humor misdirection. Am J Ophthalmol. 2010;150(1):82-87. e1.

20. Zhou C, Qian S, Yao J, et al. Clinical analysis of 50 Chinese patients with aqueous misdirection syndrome: a retrospective hospital-based study. J Int Med Res. 2012;40(4):1568-1579.

21. Tsai JC, Khaw PT, Hitchings RA. Management of pseudophakic malignant glaucoma. Ophthalmology. 2002;109(5):820-821. [author reply].

22. Sharma A, Sii F, Shah P, Kirkby GR. Vitrectomy-phacoemulsificationvitrectomy for the management of aqueous misdirection syndromes in phakic eyes. Ophthalmology. 2006;113(11):1968-1973.

23. Luntz MH, Rosenblatt M. Malignant glaucoma. Surv Ophthalmol. 1987; 32(2):73-93.

24. Jarade EF, Dirani A, Jabbour E, Antoun J, Tomey KF. Spontaneous simultaneous bilateral malignant glaucoma of a patient with no antecedent history of medical or surgical eye diseases. Clin Ophthalmol.2014; 8:1047-1050.

25. Jonas JB, Berenshtein E, Holbach L. Anatomic relationship between lamina cribrosa, intraocular space, and cerebrospinal fluid space. Invest Ophthalmol Vis Sci. 2003;44(12):5189-5195.

26. Killer HE, Jaggi GP, Flammer J, Miller NR, Huber AR, Mironov A. Cerebrospinal fluid dynamics between the intracranial and the subarachnoid space of the optic nerve. Is it always bidirectional? Brain. 2007;130(pt 2): 514-520.

27. Pescosolido N, Cavallotti C, Rusciano D, Nebbioso M. Trabecular meshwork in normal and pathological eyes. Ultrastruct Pathol. 2012; 36(2):102-107.
Clinical Ophthalmology

\section{Publish your work in this journal}

Clinical Ophthalmology is an international, peer-reviewed journal covering all subspecialties within ophthalmology. Key topics include: Optometry; Visual science; Pharmacology and drug therapy in eye diseases; Basic Sciences; Primary and Secondary eye care; Patient Safety and Quality of Care Improvements. This journal is indexed on Submit your manuscript here: http://www.dovepress.com/clinical-ophthalmology-journal

\section{Dovepress}

PubMed Central and CAS, and is the official journal of The Society of Clinical Ophthalmology (SCO). The manuscript management system is completely online and includes a very quick and fair peer-review system, which is all easy to use. Visit http://www.dovepress.com/ testimonials.php to read real quotes from published authors. 